\title{
Intraductal Proliferative Lesions of the Breast-Terminology and Biology Matter: Premalignant Lesions or Preinvasive Cancer?
}

\author{
Leopoldo Costarelli, ${ }^{1}$ Domenico Campagna, ${ }^{1}$ Maria Mauri, ${ }^{2}$ and Lucio Fortunato ${ }^{3}$ \\ ${ }^{1}$ Department of Pathology, San Giovanni-Addolorata Hospital, Rome, Italy \\ ${ }^{2}$ Department of Medical Oncology, San Giovanni-Addolorata Hospital, Rome, Italy \\ ${ }^{3}$ Department of Surgery, San Giovanni-Addolorata Hospital, Rome, Italy
}

Correspondence should be addressed to Leopoldo Costarelli, lcostarelli@hotmail.com

Received 22 December 2011; Accepted 21 February 2012

Academic Editor: Virgilio Sacchini

Copyright (c) 2012 Leopoldo Costarelli et al. This is an open access article distributed under the Creative Commons Attribution License, which permits unrestricted use, distribution, and reproduction in any medium, provided the original work is properly cited.

Morphological criteria for the diagnosis of intraductal proliferative lesions of the breast have been an object of research and much controversy, and its terminology is rather confusing. Knowledge of the molecular aspects of this disease probably necessitates further research to clarify if these entities can be identified as breast cancer precursors or as a malignant preinvasive disease. These issues are of great interest not only for their biological implications, but also to the clinician who must understand the disease and direct therapies. Molecular studies have shown that epitheliosis (usual ductal hyperplasia) is not monoclonal, while malignant lesions (atypical ductal hyperplasia, flat epithelial atypia, low-grade and high-grade intraductal carcinoma) constantly show these characteristics. These malignant lesions, classified with a DIN grading system (ductal intraepithelial neoplasia), are not obligate precursors of invasive ductal carcinoma and do not represent different evolving grades in a linear model of cancerogenesis. Breast cancerogenesis probably has different pathways with different morphologic precursors.

\section{Introduction}

Intraductal proliferative lesions (IPLs) of the breast are confined to the duct-lobular system, originating from the terminal duct-lobular unit (TDLU) with different cytological and architectural patterns of proliferation. They are characterized by an increase in the number of cells perpendicular to the basement membrane resulting in total alteration and distension of the normal unit structure of the breast without increasing in number [1].

Both a lobular and a ductal type of epithelial proliferation of TDLU are recognized. While the first type is quite monomorphic, intraductal proliferations show a wide variety of heterogeneous cytological aspects and architectural patterns.

In the past several decades, there has been a wide discussion among pathologists worldwide regarding classification and grading of IPLs, with the aim to establish various risk categories. Despite these efforts, a high interobserver variability among pathologists regarding the diagnosis of IPLs as well established [2].

Criteria for diagnosis of IPLs by morphological means (Hematoxylin and Eosin and immunohistochemistry stains) are both qualitative (cytological and architectural changes), and quantitative. Diagnostic variability is not only due to interpretation of different patterns, but also to the difficulty in recognizing atypical cells isolated or in small clusters in TDLU.

In this regard, there are several open questions in the literature, including the minimum threshold for grading, the risk of progression for different type of lesions, and the relationships between normal epithelium, IPLs, and invasive ductal carcinoma (IDC). Furthermore, it is unclear whether different types of IPLs are progressive steps of the same process or represent independent lesions leading to different malignancies. In the last few years, there have been numerous studies in this field, and several answers have been suggested to these questions. 
These issues are of great interest not only to those involved in basic research, but also to the clinicians, because each type of lesion has different propensity for progression to local relapse and invasive disease. Therefore, understanding the biology of these lesions is paramount, and will contribute to a better delineation of appropriate guidelines for surgical treatment, as well as adjuvant and diagnostic recommendations.

\section{Terminology and Historical Aspects}

The term utilized by Azzopardi [3] for benign epithelial hyperplasia was epitheliosis. This term found little agreement among pathologists and has gained much wider acceptance in Europe than in North America. The alternative terms in the past were papillary proliferation and papillomatosis, because it can form "tongue-like" projection into ductal lumina, but without the connective core in papillae seen in papillary lesions of other organs. To date, epitheliosis appears a correct term because it includes the various patterns of benign proliferation, as fenestrated, solid, or papillary aspects. In recent years, the term Usual Duct Hyperplasia (UDH) [4] has been utilized for these lesions.

Epitheliosis is a condition that has to be distinguished from in situ well-differentiated, low-grade carcinoma (LGDCIS). The criteria for diagnosis have been well described by Azzopardi [3]. Two cell types are distinguishable in epitheliosis, epithelial, and myoepithelial, which have divergent differentiation. Immunohistochemical stains (p63, actin, calponin) are useful to detect myoepithelial cells in the lesion (Figures 1(a) and 1(b)). In epitheliosis, there is little distension of TDLU, few calcifications (not in granular form in necrotic debris), and absence of necrosis. A particularly complex pattern is the infiltrating epitheliosis $[3,5]$, previously described as sclerosing adenosis with pseudo-infiltration [6]. The so-called sclerosing papillary proliferation of Fenoglio and Lattes [7] probably represents the same entity. The hyperplastic epithelial structures are irregulars, distorted by an elastotic and sclerotic stroma.

The hallmarks of LG-DCIS are architectural and cytological aspects. Myoepithelial cells cover the neoplastic ducts but are not present in the neoplastic proliferation (Figures $3(\mathrm{a})$ and 3(b)). Intraluminal or intraepithelial calcification, as calcific bodies, are frequent. The solid form of LGDCIS has the same cytology. Another type of LG-DCIS was referred to by Azzopardi [3] as type 2 or monomorphous clinging carcinoma (Figure 2). This pattern is particularly difficult to recognize, because the change is only cytological. One or few layers of columnar, atypical cells, define the lumen of variously larger TDLU. Pathologists have been much more reluctant to accept Azzopardi's second type of clinging carcinoma as a type of DCIS, particularly in the United States. This type of LG-DCIS is referred to also as atypical cystic lobules, atypical lobules type A, atypical columnar change, [8] and, in recent years, flat epithelial atypia (FEA) [4].

A "borderline" entity, between UDH and LG-DCIS, is controversial. Historically, there are two opposing approaches. The first viewpoint entails sharp demarcation between UDH and LG-DCIS, without intermediate lesions. Azzopardi said "...names like atypical hyperplasia should be avoided as far as possible." The second viewpoint entails the existence of a continuum between hyperplasia and LGDCIS, with different risk of progression for different grades of proliferation and atypia. Page et al. $[9,10]$ established two grades of hyperplasia, including atypical hyperplasia. Atypical hyperplasia is diagnosed when some features of LG-DCIS are present but other are lacking. When the duct is not completely involved, or when cytological appearance does not meet all the criteria of LG-DCIS. Other grading systems of IPLs suggested different grading of proliferation and atypia in borderline lesions [11]. The criteria proposed for the diagnosis of atypical ductal hyperplasia (ADH) are different: (i) lesions with cytological and architectural patterns of LG-DCIS are present, but both are not present in full flower; (ii) lesions with cytologic feature of LGDCIS but lacking the typical growth pattern; (iii) lesions with cytological and architectural patterns of LG-DCIS but measuring in aggregate less than $2 \mathrm{~mm}$ [1]. Tavassoli [4] accepted the last criteria.

Intermediate grade DCIS (IG-DCIS) and high-grade DCIS (HG-DCIS) are obvious neoplastic lesions, graded by nuclear grade, necrosis, and architectural patterns. IGDCIS has solid, papillary, or cribriform growth patterns, cytologically similar to those of LG-DCIS but with intraluminal necrosis (Figure 4(a)) or with intermediate cytological grade (Figure 4(b)), with or without necrosis. HG-DCIS has highly atypical cells proliferating as a single layer, or with papillary, cribriform, or solid pattern, usually with intraluminal necrosis. The HG-DCIS with solid pattern and large intraductal necrosis is referred to as comedocarcinoma (Figure 5). Typical granular calcifications on necrosis are present.

Rosai [2] proposed a terminology such as mammary intraepithelial neoplasia (MIN), like CIN of uterine cervix, for subjectivity and high degree of variability in interpretation of IPLs, disagreement about the criteria for definition of borderline lesions, and risk of progression of all types of IPLs. Afterwards this concept has been drawn as ductal intraepithelial neoplasia.

\section{DIN System}

Tavassoli [1] proposed to comprise all the IPLs in a single category, termed DIN, with various subtypes. This initial work by Tavassoli included epitheliosis or intraductal hyperplasia as DIN1a, because the risk of developing an invasive carcinoma was 1.5-2 times higher than in the general population. In the WHO book [4], UDH is separated by DIN, which includes FEA (DIN1A), ADH (DIN1B), LGDCIS (DIN1C), IG-DCIS (DIN2), and HG-DCIS (DIN3) (Table 1).

DIN System has some advantages. It diminishes the dualism cancer/no cancer, retains separation of all different subcategories but places LG-DCIS in the same group of $\mathrm{ADH}$, because it considers the differences between these lesions quantitative, not qualitative. It eliminates the term cancer, diminishing the associated anxiety and emotional 


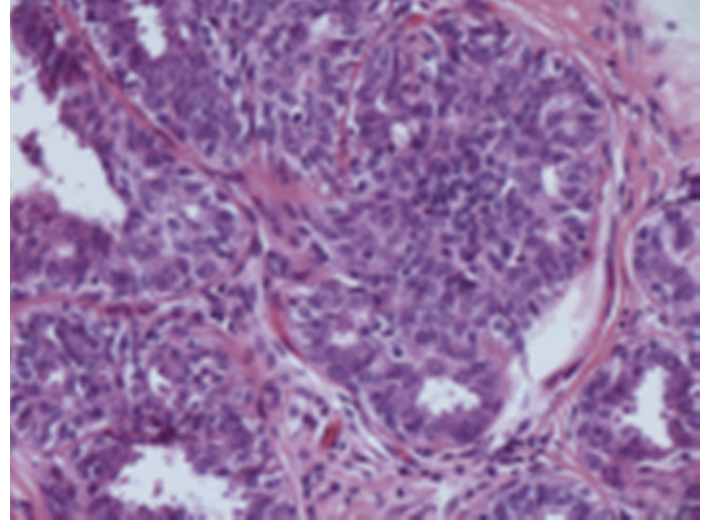

(a)

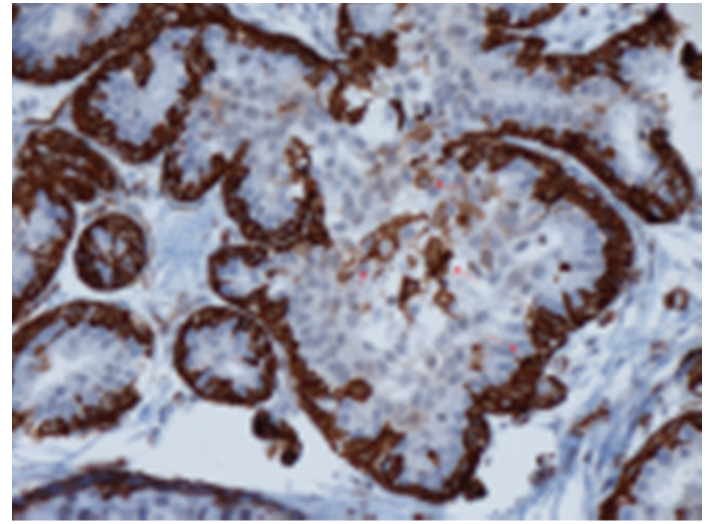

(b)

FIGURE 1: H.E. and immunoreaction for SM-actin—epitheliosis (usual ductal hyperplasia). Intraductal proliferation with irregular, "slitlike" lumina. The immunoreaction shows myoepithelial cells (arrows) surrounding the duct and in the proliferation.

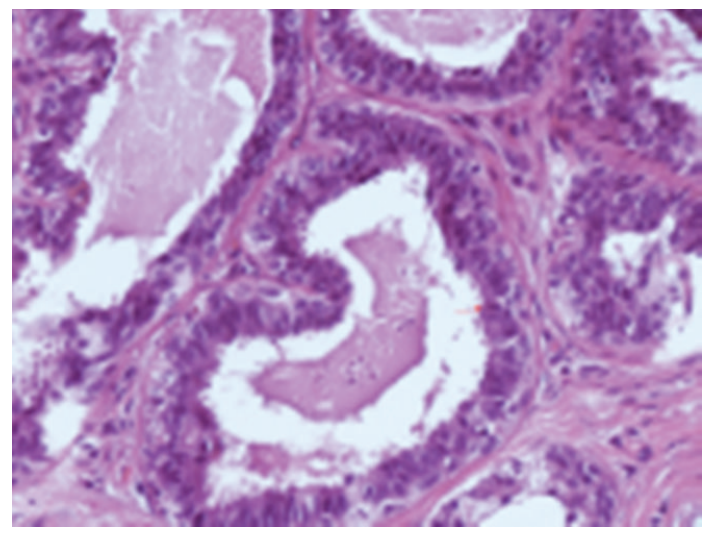

Figure 2: H.E._-flat epithelial atypia (DIN1a)_large TDLU with one to three layers of atypical ductal cells and mitosis.

stress, and it incorporates the monomorphus clinging DCIS (FEA) in the same group of ADH and LG-DCIS.

The majority of the participants in the WHO Working Group were in favour of maintaining the traditional terminology with the new DIN System [4]. This fact shows well the disagreement and scepticism of pathologists.

\section{Genetic and Molecular Findings}

In the last twenty years, there have been numerous studies to search for genetic and molecular differences or similarities between the various forms of IPLs, heterogeneous in their cytological and architectural characteristics, and between IPLs and normal TDLU and IDC. Sometimes the results seem conflicting, but a more careful analysis reveals very interesting information about histogenesis, evolution, degree of progression, and invasiveness. The apparent contradictions are related to the different methods employed, with the difficulty of performing studies on very small lesions, with the possibility of contamination by normal tissue around the lesion. Laser capture microdissection has reduced these problems, and the possibility of using very advanced methods like comparative genomic hybridization on paraffin-embedded tissues has brought to utilize archive material [12].

A multitude of methods have been utilized: immunohistochemistry (IHC), in situ hibridization (ISH), analysis of loss of heterozygosity and allelic imbalance (AI), Comparative Genomic hybridization (CGH), cDNA microarrays (MA), and Proteomics Analysis (PA). The purposes of these methods are the study of growth characteristics, the expression of oncogenes, tumor suppressor genes, and other molecules, comparison of $\mathrm{LOH}$ and $\mathrm{AI}$ between the various forms of IPLs, and IPLs versus normal TDLU, IPLs versus IDC.

4.1. Growth Characteristics. The growth of any hyperplastic or neoplastic lesion is a balance between proliferation and cell death (apoptosis). Many researchers have studied the Proliferation Index (PI) with IHC, using antibodies marking cellular proliferative cycle like Ki67 [13]. In premenopausal women, the PI of the normal TDLU varies in different phases of the menstrual cycle. In luteal phase, it is higher than in proliferative phase. The median PI in normal TDLU is $2 \%$, in $\mathrm{ADH}$ it is 5\%, in DCIS it is 5\%. LG-DCIS and FEA have a median PI of $5 \%$, while in HG-DCIS it is $20 \%$. In any case there is a continuous range from $1 \%$ and $70 \%$ from very well and poorly differentiated lesions.

$\mathrm{ADH}$ has a lower rate of apoptosis $(0,3 \%$ versus $0,6 \%)$ and higher PI than normal TDLU; DCIS has higher apoptosis (5\%) and PI than normal TDLU and ADH. Apoptosis varies from $1 \%$ to $5 \%$ among LG-DCIS and HG-DCIS, and it represents a continuous variable [14]. Higher cellular death in high-grade lesions means that the growth is a complex phenomenon. HG-DCIS has large positive growth imbalance with a high cellular death. Disturbance of the equilibrium between cell proliferation and cell death is the result of numerous alterations of growth regulating mechanisms involving sex hormones, oncogenes, and tumor suppressor genes. 


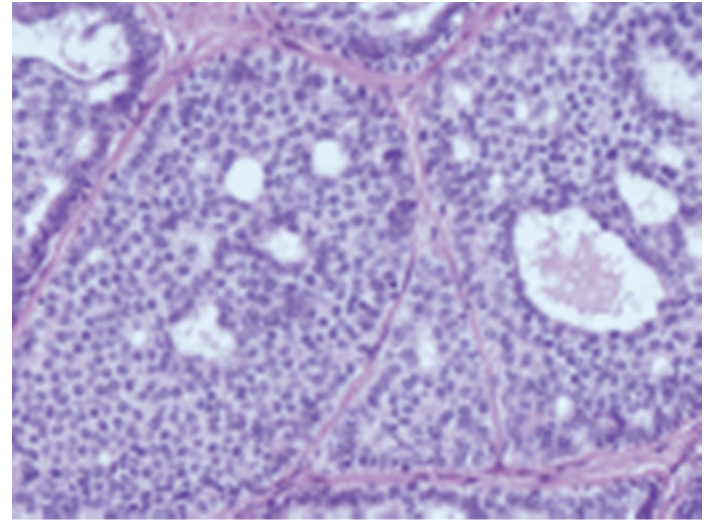

(a)

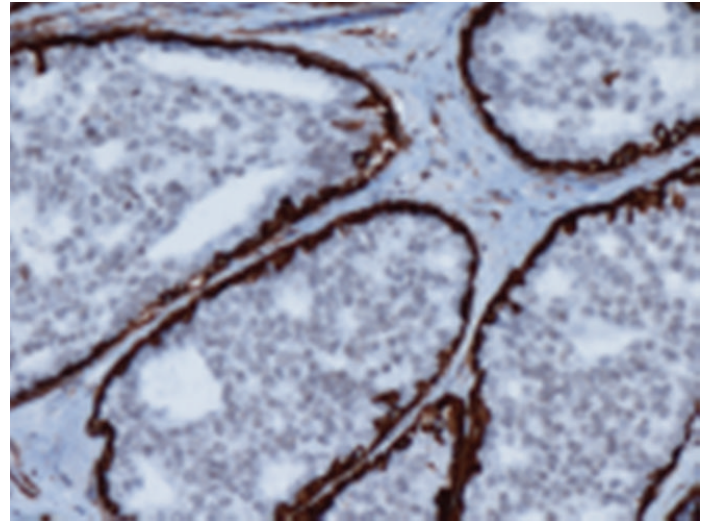

(b)

FIGURE 3: H.E and immunoreaction for SM-actin-low grade ductal in situ carcinoma (DIN1c). Cribriform type of intraductal proliferation with round, regular lumina, monomorphic round nuclei. Myoepithelial cells surround the duct but are not in the proliferation.

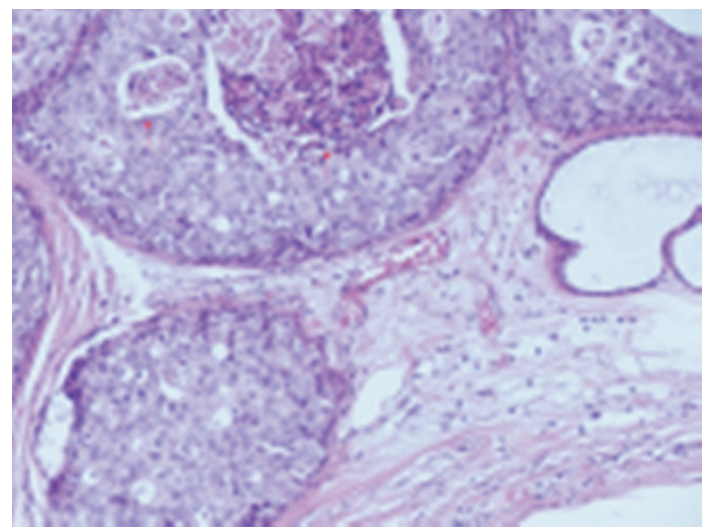

(a)

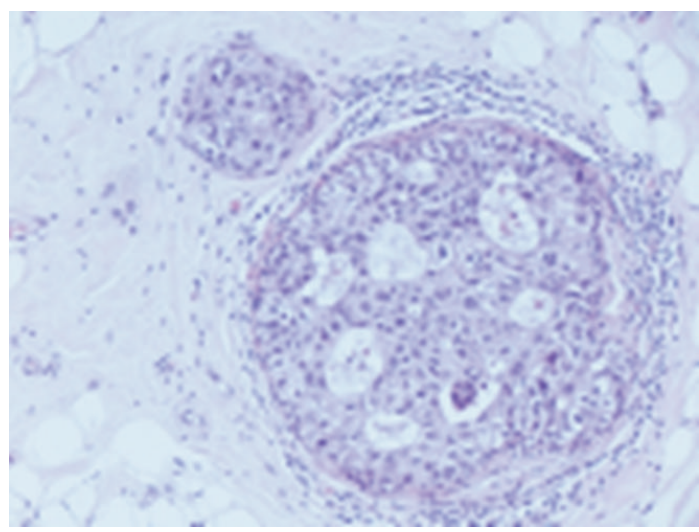

(b)

FIgURE 4: H.E.-intermediate grade ductal in situ carcinoma (DIN2). Obvious cribriform ductal in situ carcinoma with necrosis (Figure 4(a)—arrow) and intermediate cytological grade.

4.2. Sex Hormones, Oncogenes, and Tumor Suppressor Genes. Estrogens, through Estrogen Receptor (ER), play a central role in growth and differentiation of normal TDLU epithelium, stimulating cell proliferation and regulating expression of other genes, like Progesteron Receptor (PgR) [15]. Two types of ER are described: $\mathrm{ER} \alpha$ and $\mathrm{ER} \beta$. ER $\alpha$ is the more studied molecule. $90 \%$ of normal TDLU express ER in an average $30 \%$ of cells [14]. There is a change of expression during the menstrual cycle: in the luteal phase, the rate of positive cells is higher (40\% versus 20\%). In postmenopausal women, the rate is higher $(50 \%)$. Nearly all cells of ADH express ER. ER is expressed in 75\% of DCIS; $100 \%$ in LGDCIS, nearly all cells, and $30 \%$ in HG-DCIS, usually in a rate of cells $[14,16]$.

Many molecules are studied in IPLs, but the majority of studies have not been validated [17]. Exceptions are c-erbB2 (neu) and p53.

In IDC, c-erbB2 (neu) is overexpressed or amplified in $10-20 \%$ of cases, generally HG-IDC. It plays a role in cell proliferation, is related to poor clinical outcome, is a predictive marker for responsiveness to various therapies, and promotes cell mobility [18]. In recent years, it has been one of the more studied markers in breast cancer, because it is a target for trastuzumab therapy. Normal TDLU, UDH, $\mathrm{ADH}$, and FEA do not express c-erbB2 (neu). LG-DCIS and intermediate grade express neu in less than $10 \%$ of cases, whereas in HG-DCIS it is overexpressed in $60 \%$ of cases [19].

The more utilized method to detect expression of p53 is IHC, which is a surrogate assay for detecting mutations, because a gene with missense mutations codifies for inactivate protein. This abnormal inactive protein is accumulated in very high levels in the nucleus of neoplastic cells, and it is detectable by IHC [20]. 30\% of IDC overexpress p53, and it is related to aggressive biological features and poor clinical outcome. Normal TDLU, UDH, and $\mathrm{ADH}$ do not overexpress p53, apart from in the Li-Fraumeni syndrome, characterized by inherited mutations. In DCIS p53 correlates 


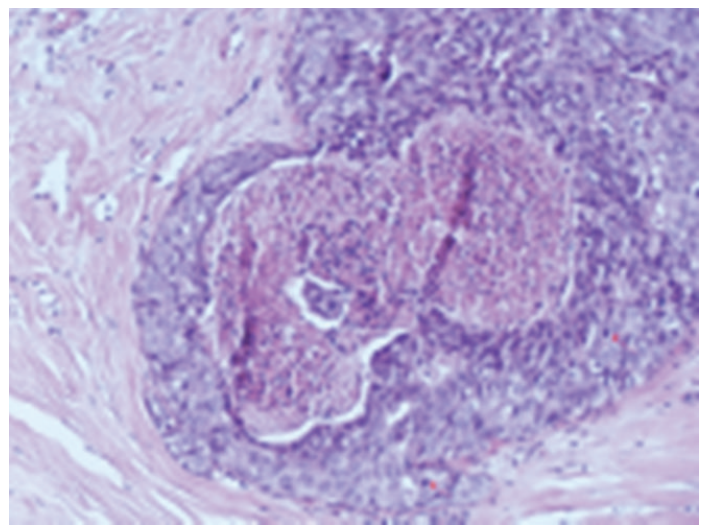

FIGURE 5: H.E.- -high-grade ductal in situ carcinoma (DIN3). Solid intraductal carcinoma with high cytological grade and necrosis (comedocarcinoma) and numerous mitosis.

with differentiation. It is rare in LG-DCIS and common (40\%) in HG-DCIS [21].

Clark et al. [22] found by IHC analysis on tissue microarray (TMA) the same molecular classes in DCIS as in IDC $[23,24]$, but with different frequency. Basal-like phenotype is rare in DCIS; c-erbB2 is more frequently expressed in Luminal-like DCIS than in IDC $(13,2 \%$ versus $5,2 \%)$. The mitochondrial antiapoptotic protein $b c l 2$ is up-regulated in LG (G1-G2) Luminal-type DCIS. The same results are in the study of Tamimi et al. [25]. Livasy et al. [26] found a higher frequency of Basal phenotype in DCIS.

Other genes and proteins are studied in IPLs and compared with IDC.

Ma et al. [27] studied five genes with quantitative RTPCR in ADH and DCIS, upregulated or downregulated in IDC. These genes were altered in the same rate in $\mathrm{ADH}$, DCIS, and IDC: for example, CRIP1 was upregulated in $7 / 8 \mathrm{ADH}$, in 27/30 DCIS and in 23/25 IDC, and ELF5 was downregulated in 7/8 ADH, in 28/30 DCIS and 25/25 IDC. Significant alterations in gene expression of ADH are maintained in the later stages of DCIS and IDC. Furthermore, to characterize the molecular link between DCIS and IDC, the study recognizes other clusters of genes related to infiltrative potential. These genes establish different patterns of gene expression among DCIS, which reflect different invasive potential, and there is a gene signature of DCIS, like for IDC [28].

Gillett et al. [29] studied expression of Cyclin D1 in ADH and DCIS. In IDC, expression of Cyclin D1 is more frequent in well differentiated and ER+ cases. ADH does not express Cyclin D1, but it is ER+ like LG-DCIS: in DCIS Cyclin D1 is over-expressed in $64 \%$ of cases, and it is not related to grade or to ER expression.

Schuetz et al. [30] studied genes of epithelial mesenchymal Transition (EMT) and other genes candidates to cause invasion and metastases [31]. These genes (Twist1, SPARC, MMP13, MMP11, BPAG1) are markers of transition from DCIS to IDC. Some of the proteins codified by these genes are very interesting: for example, BPAG1 is expressed in hemidesmosomes connecting epithelial cells to basement
TABLE 1: Terminology of IPLs.

\begin{tabular}{|c|c|}
\hline DIN system & Traditional terminology \\
\hline $\begin{array}{l}\text { Usual ductal } \\
\text { hyperplasia } \\
\text { (UDH) }\end{array}$ & $\begin{array}{c}\text { Epitheliosis } \\
\text { Infiltrating epitheliosis } \\
\text { Papillomatosis } \\
\text { Sclerosing adenosis with pseudoinfiltration } \\
\text { Sclerosing papillary proliferation }\end{array}$ \\
\hline DIN1a & $\begin{array}{c}\text { Clinging carcinoma monomorphus or type } 2 \\
\text { Flat epithelial atypia } \\
\text { Atypical cystic lobules } \\
\text { Atypical lobules type A } \\
\text { Atypical columnar change }\end{array}$ \\
\hline DIN1b & Atypical ductal hyperplasia (ADH) \\
\hline DIN1c & $\begin{array}{l}\text { Low-grade ductal carcinoma in situ (LG-DCIS) } \\
\text { DCIS grade } 1\end{array}$ \\
\hline DIN2 & $\begin{array}{c}\text { Intermediate-grade ductal carcinoma in situ } \\
\text { (IG-DCIS) } \\
\text { DCIS grade } 2\end{array}$ \\
\hline DIN3 & $\begin{array}{c}\text { High-grade ductal carcinoma in situ (HG-DCIS) } \\
\text { DCIS grade } 3\end{array}$ \\
\hline
\end{tabular}

membrane. IDC cells do not contain hemidesmosomes and BPAG1 is downregulated. Matrix metalloproteinases (MMPs) can degrade different components of extracellular matrix, including laminin, fibronectin, collagen, and elastin, and are upregulated in HG-DCIS.

Porter et al. [32] and Kretschmer et al. [33] found several genes of IDC up-regulated in DCIS using SAGE (Serial Analysis of Gene Expression), TMA, quantitative RTPCR, and IHC. Some of these genes are not related to differentiation grade (MUC1, SBP1) and probably play a role in early cancerogenesis. Other proteins, like the psoriasin (S-100A7), a calcium-binding protein that regulates cell cytoskeleton and motility, are present in comedo-type HGDCIS.

4.3. $\mathrm{LOH}$ and Allelic Imbalance (AI). Two types of studies are performed: the first on IPLs in their pure form and the second on IPLs with synchronous IDC in the same breast. The strategy of the last type is to identify alterations, which may be important in the invasiveness [17]. Generally, IPLs with synchronous invasive cancer share more frequent genetic alterations with IDC than pure forms. For example, a marker on chromosome 11p (D11S988) is more frequent in all IPLs close to IDC than in IPLs without cancer [34]: morphologically normal TDLU close to IDC also shared rarely some LOH with cancer [35]. O'Connell et al. [34], assessing LOH to 15 loci on 12 chromosomes, found that $50 \%$ of $\mathrm{ADH}$ shared their $\mathrm{LOH}$ phenotypes with synchronous IDC, providing novel and compelling genetic evidence that ADH is a direct precursor of IDC. Many studies of DCIS have shown that nearly all lesions share several identical AI with synchronous IDC, providing convincing if not surprising evidence that they are evolutionarily related too [34-38]. Synchronous DCIS and IDC may occasionally 
show distinct AIs, suggesting that there may also be divergent aspects to their evolution [25].

There are numerous studies on LOH in IPLs without invasive cancer.

Allelic Imbalance in UDH is rare: Lakhani et al. [39] found alterations in $0-13 \%$ of studied loci, frequently in $17 \mathrm{q}$; Deng et al. [35] found alterations in different loci in $0-15 \%$.

Moinfar et al. [40] found AI on 77\% of FEA, at least in one locus $11 \mathrm{q}, 16 \mathrm{q}$, and $3 \mathrm{p} .11 \mathrm{q}$ and $16 \mathrm{q}$ are frequently involved in tubular carcinoma.

Morphological overlap between ADH and LG-DCIS is reflected at molecular level. Up to $50 \%$ of ADH contains one or more AIs among 30 genetic loci $[35,39]$, and they are the same of DCIS.

CGH analysis of DCIS has demonstrated a large number of alterations, including gains of $1 \mathrm{q}, 5 \mathrm{p}, 6 \mathrm{q}, 8 \mathrm{q}, 17 \mathrm{q}, 19 \mathrm{q}$, 20p, 20q, and Xq, and losses of 2q, 5q, 6q, 8p, 9p, 11q, 13q, $14 q, 16 q, 17 p, 17 q$, and 22q [35, 41, 42]. Some AI are more frequent and constitute hot spots: loss in 11q, 16q, 17p and $17 \mathrm{q}$.

Rosenberg et al. [43] studied a series of 15 microsatellite loci in $\mathrm{ADH}$ and found monoclonal microsatellite alterations in $40 \%$ of cases in more than one locus, suggesting that a genetic instability plays an early role in cancer progression.

Wiechmann and Kuerer [44] characterize the differences between LG-DCIS and HG-DCIS and their risk of progression in IDC by means of the expression of steroid receptor (LG-DCIS is frequently ER/PgR positive), growth characteristics (Ki67 is lower in LG-DCIS than in HGDCIS), expression of c-erbB2 (frequent in HG-DCIS, rare in LG-DCIS), bcl2 and p53 (the first over-expressed in LG-DCIS, the second frequently mutated in HG-DCIS), expression of psoriasin (S-100A7) and metalloproteinases (MMPs) (upregulated in HG-DCIS), and allelic imbalance (LG-DCIS has frequently gain of $1 \mathrm{q}$ and loss of $16 \mathrm{q}$, whereas HG-DCIS shows frequently $17 \mathrm{q} 12$ and $11 \mathrm{q} 13$ amplification).

In Table 2 are reported the more frequent alterations in IPLs and IDC.

\section{Discussion}

One of the most controversial topics about breast pathology concerns IPL, with a wide range of phenotypic manifestations from epitheliosis to DCIS. Page et al. $[9,10]$ have suggested that there is a continuum between these two extremes, with an intermediate condition called ADH. Azzopardi [3], on the other hand, draws a sharp line of demarcation between hyperplastic and neoplastic lesions, and he stigmatizes: "...names like "atypical hyperplasia" should be avoided as far as possible". He himself describes a particular type of DCIS called clinging carcinoma. The lowgrade clinging carcinoma (type 2 according to Azzopardi) and columnar change is named also flat epithelial atypia (FEA).

High interobserver variability among experienced pathologists in $\mathrm{ADH}$ interpretation is reported [2], mainly related to different proposed criteria. WHO book [4] suggests the use of dimensional criteria of Tavassoli [1]:
TABLE 2: "hot spots" in IPLs-more frequent allelic imbalances reported.

\begin{tabular}{lcc}
\hline & Gains & Losses \\
\hline UDH & rare & rare \\
ADH & $1 \mathrm{q}, 16 \mathrm{p}$ & $11 \mathrm{p}, 11 \mathrm{q}, 16 \mathrm{q}, 17 \mathrm{p}$ \\
FEA & - & $3 \mathrm{p}, 11 \mathrm{q}, 16 \mathrm{q}$ \\
& $1 \mathrm{q}(L G-D C I S), 5 \mathrm{p}$, & $2 \mathrm{q}, 5 \mathrm{q}, 6 \mathrm{q}, 8 \mathrm{p}, 9 \mathrm{q}, 11 \mathrm{q}$ \\
& $6 \mathrm{q}, 8 \mathrm{q}, 17 \mathrm{q}, 19 \mathrm{q}$, & $(H G-D C I S), 13 \mathrm{q}(H G-D C I S), 14 \mathrm{q}$, \\
DCIS & $20 \mathrm{p}, 20 \mathrm{q}$ & $16 \mathrm{q}(L G-D C I S), 17 \mathrm{p}, 17 \mathrm{q}$ \\
& $(H G-D C I S), \mathrm{Xq}$ & $(H G-D C I S), 22 \mathrm{q}$ \\
\hline
\end{tabular}

$\mathrm{ADH}$ is a lesion with cytological and architectural pattern of LG-DCIS measuring in aggregate less than $2 \mathrm{~mm}$.

The various IPLs have different risk of progression: UDC has two folds, ADH four folds, and LG-DCIS ten folds compared to normal breast $[9,10]$. At the molecular level, rarely UDH shows allelic imbalance (AI) for one gene, whereas ADH, FEA, and LG-DCIS show frequent AIs for many genes [34-39]. Expression of high-molecularweight cytokeratin is different between UDH and other IPLs: UDH consistently displayed the presence of a population of cytokeratin 5/6-expressing basal-type cells within the proliferative lesion, whereas ADH and LG-DCIS lacked cytokeratin 5/6-positive cells. A subset of HG-DCIS express cytokeratin 5/6: it is probably the precursor of Basal-like IDC [42]. The studies about growth characteristics (proliferative index and apoptosis) and about expression of different molecules like ER, oncogenes, and tumor suppressor genes display a substantial difference between UDH and other IPLs. ADH, FEA, and LG-DCIS, on the other hand, shares many biological characteristics with IDC $[13,14,16,17,45]$, and microsatellite analysis shows monoclonality and genetic instability in ADH [43]. This supports the concept that UDH is a not malignant lesion, the opposite to other IPLs. ADH, FEA and LG-DCIS can be set in the same group of preinvasive breast neoplastic lesions. DIN system, as well as proposed by Tavassoli [4], includes ADH, FEA and DCIS, not UDH.

Several data support the concept that different types of DCIS show different genetic alterations [41, 42]. Alterations at $16 \mathrm{q}$ are much more frequent in LG-DCIS than in HGDCIS, in which alterations at $13 \mathrm{q}, 17 \mathrm{q}$, and $20 \mathrm{q}$ are more frequent $[4,6,7,10]$. Similar findings are in invasive carcinomas of low and high grade [41, 42, 46-48]. On the other hand, LG-DCIS share many molecular alterations with ADH $[35,39]$ and LG-IDC, and also the few studies on FEA [40] show alterations similar to LG-IDC, in particular with a very well-differentiated IDC (tubular carcinoma). These molecular studies reflect the same morphological findings: (i) it is extremely rare to find an HG-DCIS in a LGIDC, as well as a LG-DCIS in an HG-IDC; (ii) in tubular carcinoma we see frequently an in situ component like FEA or LG-DCIS cribriform type. Also, growth characteristics and rates of expression of sex hormones, oncogenes, and tumor suppressor genes suggest that LG-DCIS is a precursor of LG-IDC and HG-DCIS is a precursor of HG-IDC [41, 42]. 
TABLe 3: Cancerogenesis models.

Linear model of progression

TDLU $\Rightarrow$ UDH $\Rightarrow$ ADH or FEA $\Rightarrow$ LG-DCIS $\Rightarrow$ HG-DCIS $\Rightarrow$ IDC $\Rightarrow$ Metastases

Parallel model of progression

(1) TDLU $\Rightarrow$ ADH $\Rightarrow$ LG-DCIS $\Rightarrow$ LG-IDC $\Rightarrow$ Metastases

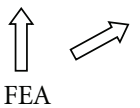

(2) TDLU $\Rightarrow$ HG-DCIS $\Rightarrow$ HG-IDC $\Rightarrow$ Metastases

TABLE 4: Classification of DCIS based on biologic potential [44].

\begin{tabular}{|c|c|c|c|c|c|}
\hline \multirow{3}{*}{$\begin{array}{c}\text { Ductal } \\
\text { cell }\end{array}$} & \multirow{3}{*}{$\Rightarrow$} & \multirow{3}{*}{$\begin{array}{l}\text { Dysplastic } \\
\text { cell (?) }\end{array}$} & \multirow{2}{*}{$\begin{array}{l}\text { LG-DCIS } \\
\text { Histology: } \\
\text { - Low nuclear grade } \\
\text { - ER/PgR positive } \\
\text { - No comedo necrosis } \\
\text { Molecular markers: } \\
\text { - c-erbB2 negative } \\
\text { - Low Ki67 } \\
\text { - p53 normal } \\
\text { - bcl2 upregulated }\end{array}$} & \multirow[t]{2}{*}{$\Rightarrow$} & $\begin{array}{l}\text { Well differentiated } \\
\text { IDC }\end{array}$ \\
\hline & & & & & \\
\hline & & & $\begin{array}{l}\text { HG-DCIS } \\
\text { Histology: } \\
\text { - High nuclear grade } \\
\text { - ER/PgR negative } \\
\text { - Comedo necrosis } \\
\text { Molecular markers: } \\
\text { - c-erbB2 positive } \\
\text { - High Ki67 } \\
\text { - p53 mutations } \\
\text { - LOH 11q13 } \\
\text { - Psoriasin (S-100A7) and MMPs upregulated }\end{array}$ & & $\begin{array}{c}\text { Poorly } \\
\text { differentiated IDC }\end{array}$ \\
\hline
\end{tabular}

These data suggest that there may be multiple pathways for the evolution of IPLs and IDC. In various tissues, a linear multistep progression between various preinvasive stages, which end in invasive cancer, is recognizable: in colonic mucosa, for example, there is a linear multistep model from normal epithelium to invasive carcinoma through the sequence hyperproliferative epithelium, adenoma, carcinoma, and any morphological step is related to a specific genetic alteration [49]. In breast cancer, the linear model undoubtedly oversimplifies a complex process. There is no morphological or molecular evidence that LG-DCIS progress into an HG-DCIS, or into HG-IDC. The model that results from morphological and molecular data is horizontal (or parallel), and it is done by two or several pathways (Table 3 ).

For this reason, the DIN system is not a progression through different grades, like the intraepithelial neoplasia in other tissue (for example, the CIN system of cervical cancer), but a classification of different intraductal neoplastic conditions, each of these are not an obligate precursor of IDC.

The DCIS classification of Wiechmann and Kuerer [44], based on biological potential to progress into IDC, shows two parallel pathways of progression with different histologic characteristics and molecular markers. Between these two pathways, there is a presumptive common progenitor, a dysplastic cell, not better characterized (Table 4).

In IDC, recent studies have led to a molecular classification based on the biological characteristics of the tumor rather than limited to morphological analysis. Perou [23] and Sotiriou et al. [24] identified molecular subtypes of invasive breast cancer based on an intrinsic gene signature. Many studies have aimed to identify an IHC profile that can act as a surrogate for gene array analysis, and it appears that a five-marker panel of estrogen receptor (ER), progesterone receptor (PR), c-erbB2, cytokeratin 5/6, and EGFR shows ability to categorise invasive cancers to their molecular subtype [50]. Much less attention has been focused on dissecting the biological subtypes of DCIS, and there are discrepancies in the results. Thus, whereas several studies report the existence of a basal subtype of DCIS [25, 51], one gene array study found no firm evidence of this category of DCIS or it is much less frequent [51]. There are also other discrepancies in the relative frequency of subtypes between the in situ and invasive disease. It has been recognized, for example, that there is a higher frequency of c-erbB2-positive DCIS compared with c-erbB2-positive IDC [52]. The difference in expression of c-erbB2 is actually inexplicable: the hypotheses advanced are that the expression 
is switched off during invasion or that many c-erbB2-positive DCIS do not transform to IDC.

\section{Conclusions}

Diagnosis and reproducibility of proposed criteria of IPLs are complex. This is testified by terminological confusion, with a large number of designations for the same entity. To render the issue even more controversial, it is not clear whether some of these entities really occur in practice. Interobserver agreement in diagnosis of intermediate lesions is low. The DIN system unifies the terminology, while it may have the additional advantage to decrease the anxiety and emotional stress of patients.

Morphological complexity is reflected by the large variety of molecular findings described by a number of studies in the last twenty years. Some of these alterations are confirmed by different studies, and their comparison with different clinical entities provides much information about their nature and propensity for progression. UDH or epitheliosis is probably a benign process, while other IPLs (ADH, LG-DCIS, HGDCIS, FEA) are neoplastic processes. ADH and FEA shares many alterations with LG-DCIS and LG-IDC, while HGDCIS share many alterations with HG-IDC. Probably, the model of breast carcinogenesis is more complex than in other tissues, because the results of the molecular studies suggest parallel different pathways of carcinogenesis. By this point of view, the DIN system is not a progression through different additional steps, like the intraepithelial neoplasia in other tissues (e.g., the CIN system for uterine cervix cancer), but a classification of different conditions, which are not obligate precursors of IDC.

\section{References}

[1] F. A. Tavassoli, Pathology of the Breast, McGraw-Hill, 2nd edition, 1999.

[2] J. Rosai, "Borderline epithelial lesions of the breast," American Journal of Surgical Pathology, vol. 15, no. 3, pp. 209-221, 1991.

[3] J. G. Azzopardi, Problems in Breast Pathology, Saunders, 1979.

[4] F. A. Tavassoli, Tumors of the Breast and Female Genital Organs, IARC/WHO, 2003.

[5] V. Eusebi and R. R. Millis, "Epitheliosis, infiltrating epitheliosis, and radial scar," Seminars in Diagnostic Pathology, vol. 27, no. 1, pp. 5-12, 2010.

[6] R. W. McDivitt, F. W. Stewart et al., Tumors of the Breast, AFIP, 1998.

[7] C. Fenoglio and R. Lattes, "Sclerosing papillary proliferations in the female breast. A benign lesion often mistaken for carcinoma," Cancer, vol. 33, no. 3, pp. 691-700, 1974.

[8] F. A. Tavassoli, "Mammary intraepithelial neoplasia: a translational classification system for the intraductal epithelial proliferations," Breast Journal, vol. 3, no. 1, pp. 48-58, 1997.

[9] D. L. Page and T. J. Anderson, Diagnostic Histopathology of the Breast, Churchill Livingstone, 1987.

[10] D. L. Page, W. D. Dupont, L. W. Rogers, and M. S. Rados, "Atypical hyperplastic lesions of the female breast. A longterm follow-up study," Cancer, vol. 55, no. 11, pp. 2698-2708, 1985.
[11] M. M. Black, T. H. Barclay, S. J. Cutler, B. F. Hankey, and A. J. Asire, "Association of atypical characteristics of benign breast lesions with subsequent risk of breast cancer," Cancer, vol. 29, no. 2, pp. 338-343, 1972.

[12] J. Isola, S. DeVries, L. Chu, S. Ghazvini, and F. Waldman, "Analysis of changes in DNA sequence copy number by comparative genomic hybridization in archival paraffin-embedded tumor samples," American Journal of Pathology, vol. 145, no. 6, pp. 1301-1308, 1994.

[13] D. C. Allred, P. O'Connell, S. A. W. Fuqua, and C. K. Osborne, "Immunohistochemical studies of early breast cancer evolution," Breast Cancer Research and Treatment, vol. 32, no. 1, pp. 13-18, 1994.

[14] F. C. Schmitt, "Multistep progression from an oestrogendependent growth towards an autonomous growth in breast carcinogenesis," European Journal of Cancer A, vol. 31, no. 12, pp. 2049-2052, 1995.

[15] B. E. Henderson, R. Ross, and L. Bernstein, "Estrogens as a cause of human cancer: the Richard and Hinda Rosenthal foundation award lecture," Cancer Research, vol. 48, no. 2, pp. 246-253, 1988.

[16] S. K. Mohsin, S. C. Hilsenbeck, and D. C. Allred, "Estrogen receptors and growth control in premalignant breast disease," Modern Pathology, vol. 13, pp. 28-36, 2000.

[17] D. C. Allred, S. K. Mohsin, and S. A. W. Fuqua, "Histological and biological evolution of human premalignant breast disease," Endocrine-Related Cancer, vol. 8, no. 1, pp. 47-61, 2001.

[18] C. R. De Potter, "The neu-oncogene: more than a prognostic indicator?" Human Pathology, vol. 25, no. 12, pp. 1264-1268, 1994.

[19] D. C. Allred, G. M. Clark, R. Molina et al., "Overexpression of HER-2/neu and its relationship with other prognostic factors change during the progression of in situ to invasive breast cancer," Human Pathology, vol. 23, no. 9, pp. 974-979, 1992.

[20] A. M. Davidoff, P. A. Humphrey, J. D. Iglehart, and J. R. Marks, "Genetic basis for p53 overexpression in human breast cancer," Proceedings of the National Academy of Sciences of the United States of America, vol. 88, no. 11, pp. 5006-5010, 1991.

[21] P. B. Rajan, D. J. Scott, R. H. Perry, and C. D. M. Griffith, "p53 protein expression in ductal carcinoma in situ (DCIS) of the breast," Breast Cancer Research and Treatment, vol. 42, no. 3, pp. 283-290, 1997.

[22] S. E. Clark, J. Warwick, R. Carpenter, R. L. Bowen, S. W. Duffy, and J. L. Jones, "Molecular subtyping of DCIS: heterogeneity of breast cancer reflected in pre-invasive disease," British Journal of Cancer, vol. 104, no. 1, pp. 120-127, 2011.

[23] C. M. Perou, T. Sørile, M. B. Eisen et al., "Molecular portraits of human breast tumours," Nature, vol. 406, no. 6797, pp. 747$752,2000$.

[24] C. Sotiriou, S. Y. Neo, L. M. McShane et al., "Breast cancer classification and prognosis based on gene expression profiles from a population-based study," Proceedings of the National Academy of Sciences of the United States of America, vol. 100, no. 18, pp. 10393-10398, 2003.

[25] R. M. Tamimi, H. J. Baer, J. Marotti et al., "Comparison of molecular phenotypes of ductal carcinoma in situ and invasive breast cancer," Breast Cancer Research, vol. 10, no. 4, article R67, 2008.

[26] C. A. Livasy, C. M. Perou, G. Karaca et al., "Identification of a basal-like subtype of breast ductal carcinoma in situ," Human Pathology, vol. 38, no. 2, pp. 197-204, 2007.

[27] X. J. Ma, R. Salunga, J. T. Tuggle et al., "Gene expression profiles of human breast cancer progression," Proceedings 
of the National Academy of Sciences of the United States of America, vol. 100, no. 10, pp. 5974-5979, 2003.

[28] L. J. Van't Veer, H. Dai, M. J. Van de Vijver et al., "Gene expression profiling predicts clinical outcome of breast cancer," Nature, vol. 415, no. 6871, pp. 530-536, 2002.

[29] C. E. Gillett, A. H. S. Lee, R. R. Millis, and D. M. Barnes, "Cyclin D1 and associated proteins in mammary ductal carcinoma in situ and atypical ductal hyperplasia," Journal of Pathology, vol. 184, no. 4, pp. 396-400, 1998.

[30] C. S. Schuetz, M. Bonin, S. E. Clare et al., "Progression-specific genes identified by expression profiling of matched ductal carcinomas in situ and invasive breast tumors, combining laser capture microdissection and oligonucleotide microarray analysis," Cancer Research, vol. 66, no. 10, pp. 5278-5286, 2006.

[31] G. M. Nagaraja, M. Othman, B. P. Fox et al., "Gene expression signatures and biomarkers of noninvasive and invasive breast cancer cells: comprehensive profiles by representational difference analysis, microarrays and proteomics," Oncogene, vol. 25, no. 16, pp. 2328-2338, 2006.

[32] D. Porter, J. Lahti-Domenici, A. Keshaviah et al., "Molecular markers in ductal carcinoma in situ of the breast," Molecular Cancer Research, vol. 1, no. 5, pp. 362-375, 2003.

[33] C. Kretschmer, A. Sterner-Kock, F. Siedentopf, W. Schoenegg, P. M. Schlag, and W. Kemmner, "Identification of early molecular markers for breast cancer," Molecular Cancer, vol. 10, article 15, 2011.

[34] P. O’Connell, V. Pekkel, S. A. W. Fuqua, C. K. Osborne, G. M. Clark, and D. C. Allred, "Analysis of loss of heterozygosity in 399 premalignant breast lesions at 15 genetic loci," Journal of the National Cancer Institute, vol. 90, no. 9, pp. 697-703, 1998.

[35] G. Deng, Y. Lu, G. Zlotnikov, A. D. Thor, and H. S. Smith, "Loss of heterozygosity in normal tissue adjacent to breast carcinomas," Science, vol. 274, no. 5295, pp. 2057-2059, 1996.

[36] M. R. Stratton, N. Collins, S. R. Lakhani, and J. P. Sloane, "Loss of heterozygosity in ductal carcinoma in situ of the breast," Journal of Pathology, vol. 175, no. 2, pp. 195-201, 1995.

[37] H. Fujii, R. Szumel, C. Marsh, W. Zhou, and E. Gabrielson, "Genetic progression, histological grade, and allelic loss in ductal carcinoma in situ of the breast," Cancer Research, vol. 56, no. 22, pp. 5260-5265, 1996.

[38] D. M. Radford, N. J. Phillips, K. L. Fair, J. H. Ritter, M. Holt, and H. Donis-Keller, "Allelic loss and the progression of breast cancer," Cancer Research, vol. 55, no. 22, pp. 5180-5183, 1995.

[39] S. Lakhani, N. Collins, M. R. Stratton, and J. P. Sloane, "Atypical ductal hyperplasia of the breast: clonal proliferation with loss of heterozygosity on chromosomes 16q and 17p," Journal of Clinical Pathology, vol. 48, no. 7, pp. 611-615, 1995.

[40] F. Moinfar, Y. G. Man, L. Arnould, G. L. Bratthauer, M. Ratschek, and F. A. Tavassoli, "Concurrent and independent genetic alterations in the stromal and epithelial cells of mammary carcinoma: implications for tumorigenesis," Cancer Research, vol. 60, no. 9, pp. 2562-2566, 2000.

[41] H. Buerger, F. Otterbach, R. Simon et al., "Different genetic pathways in the evolution of invasive breast cancer are associated with distinct morphological subtypes," Journal of Pathology, vol. 189, no. 4, pp. 521-526, 1999.

[42] W. Boecker, H. Buerger, K. Schmitz et al., "Ductal epithelial proliferations of the breast: a biological continuum? Comparative genomic hybridization and high-molecular-weight cytokeratin expression patterns," Journal of Pathology, vol. 195, no. 4, pp. 415-421, 2001.

[43] C. L. Rosenberg, A. De Las Morenas, K. Huang, L. A. Cupples, D. V. Faller, and P. S. Larson, "Detection of monoclonal microsatellite alterations in atypical breast hyperplasia," Journal of Clinical Investigation, vol. 98, no. 5, pp. 1095-1100, 1996.

[44] L. Wiechmann and H. M. Kuerer, "The molecular journey from ductal carcinoma in situ to invasive breast cancer," Cancer, vol. 112, no. 10, pp. 2130-2142, 2008.

[45] J. Prosser, S. G. Hilsenbeck et al., "Cell turnover (proliferation and apoptosis) in normal epithelium and premalignant lesions in the same breast," Laboratory Investigation, vol. 76, no. 1, absract 2A, p. 119, 1997.

[46] M. Aubele, A. Mattis, H. Zitzelsberger et al., "Extensive ductal carcinoma in situ with small foci of invasive ductal carcinoma: evidence of genetic resemblance by CGH," International Journal of Cancer, vol. 85, no. 1, pp. 82-86, 2000.

[47] J. S. Reis-Filho and S. R. Lakhani, "Genetic alterations in preinvasive lesions," Breast Cancer Research, vol. 5, no. 6, pp. 313319, 2003.

[48] R. Roylance, P. Gorman et al., "Allelic imbalance analysis of chromosome 16q shows that grade I and grade III invasive ductal breast cancers follow different genetic pathways," Journal of Pathology, vol. 196, no. 1, pp. 32-36, 2002.

[49] E. R. Fearon and B. Vogelstein, "A genetic model for colorectal tumorigenesis," Cell, vol. 61, no. 5, pp. 759-767, 1990.

[50] M. C. U. Cheang, D. Voduc, C. Bajdik et al., "Basallike breast cancer defined by five biomarkers has superior prognostic value than triple-negative phenotype," Clinical Cancer Research, vol. 14, no. 5, pp. 1368-1376, 2008.

[51] J. Paredes, N. Lopes, F. Milanezi, and F. C. Schmitt, "Pcadherin and cytokeratin 5: useful adjunct markers to distinguish basal-like ductal carcinomas in situ," Virchows Archiv, vol. 450, no. 1, pp. 73-80, 2007.

[52] J. Hannemann, A. Velds, J. B. G. Halfwerk, B. Kreike, J. L. Peterse, and M. J. Van de Vijver, "Classification of ductal carcinoma in situ by gene expression profiling," Breast Cancer Research, vol. 8, no. 5, article R61, 2006. 


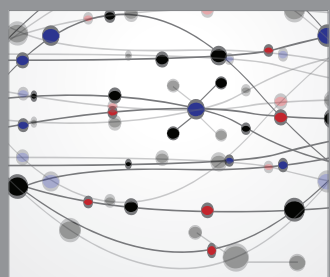

The Scientific World Journal
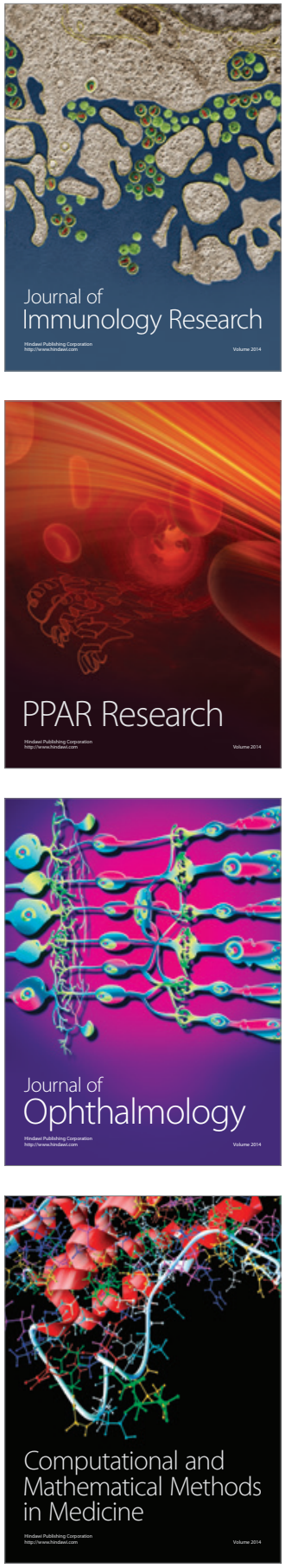

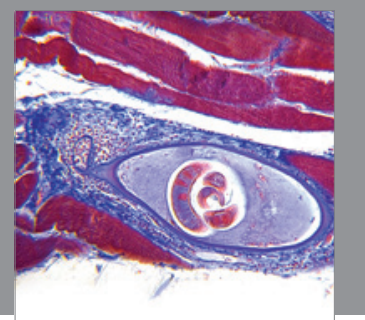

Gastroenterology

Research and Practice
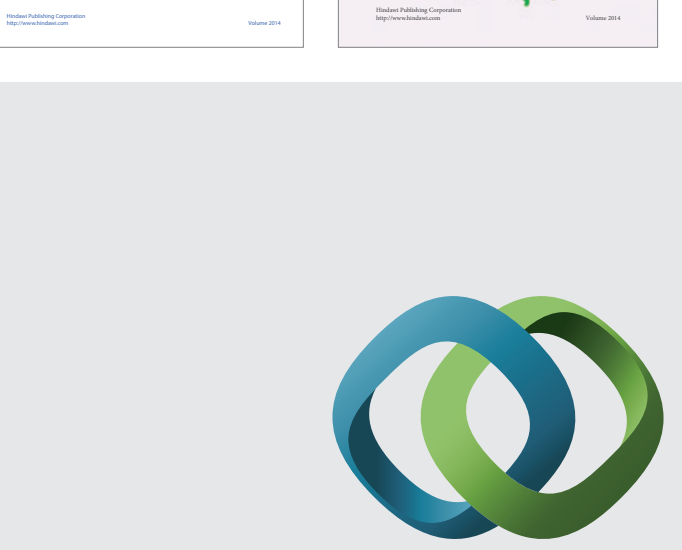

\section{Hindawi}

Submit your manuscripts at

http://www.hindawi.com
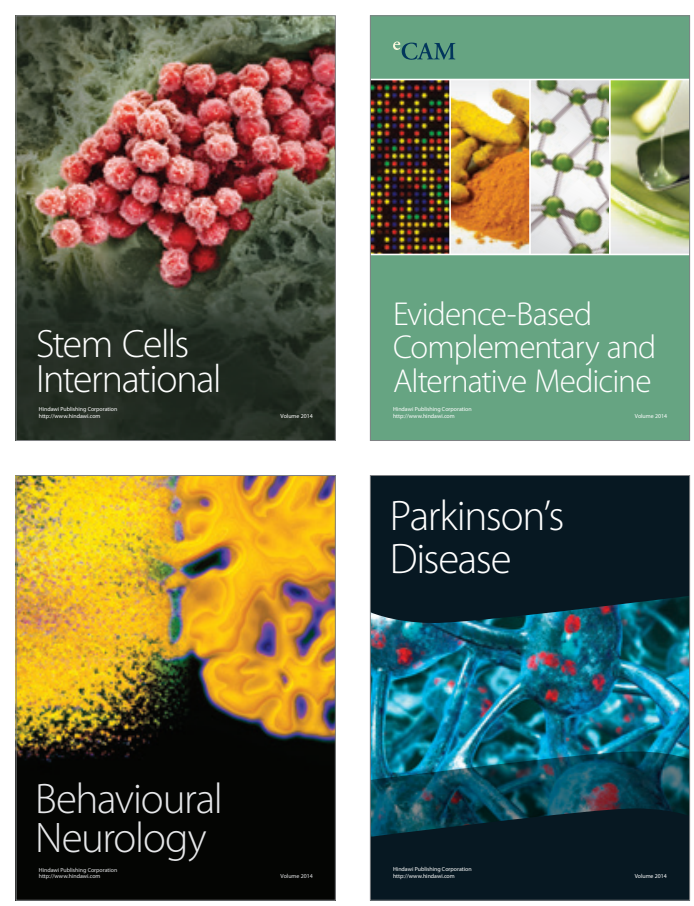

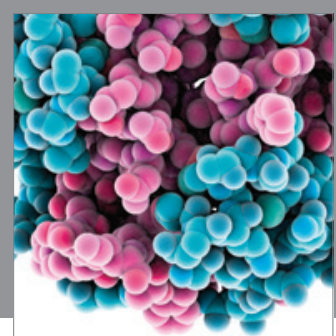

Journal of
Diabetes Research

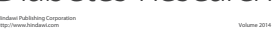

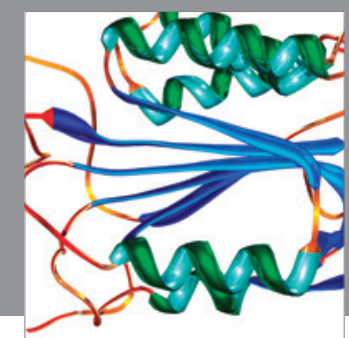

Disease Markers
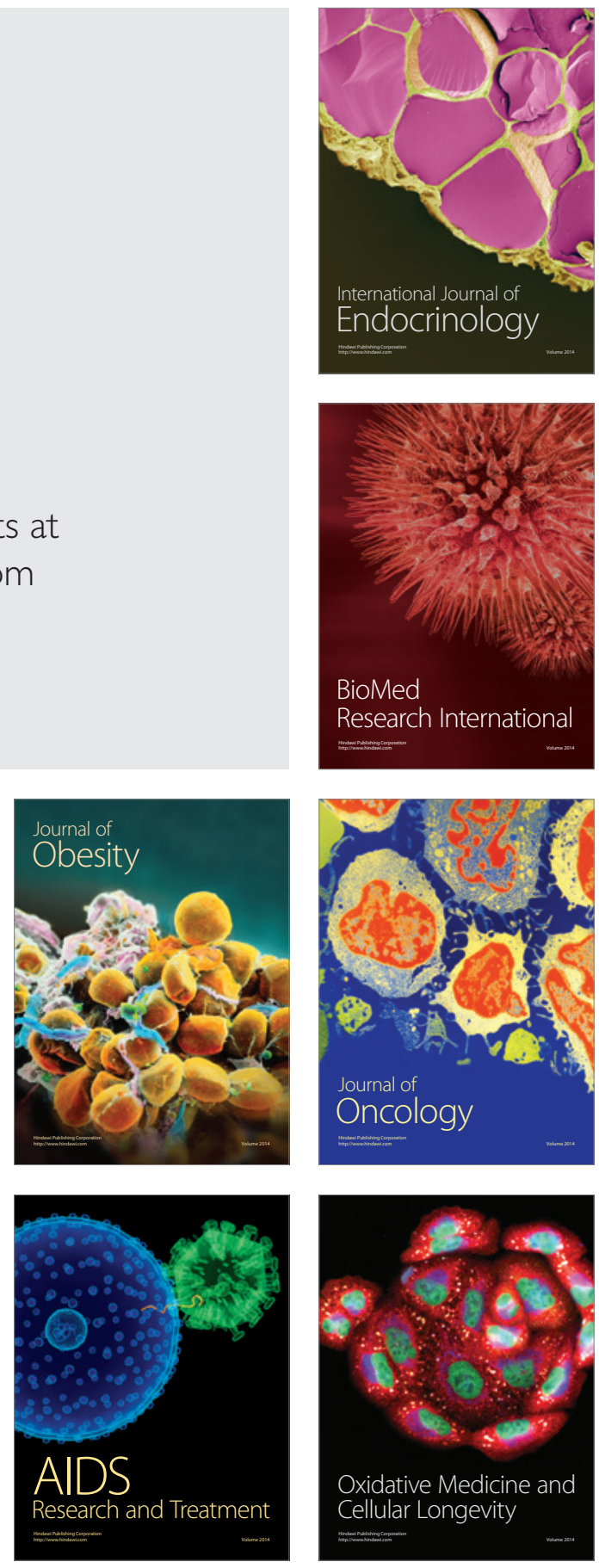